\title{
Manager- und Führungskompetenzen für Assis- tenzärzte der Allgemeinen Inneren Medizin
}

\section{Edouard Battegay}

Prof. Dr. med., im Namen des ZAIM-Stiftungsrats

Interessenkonflikte:

Als Präsident des ZAIM-Stiftungsrats bin ich massgeblich an der Gestaltung des wissenschaftlichen Programms aller ZAIMVeranstaltungen beteiligt. Weder meine Klinik noch ich persönlich beziehen Einnahmen aus dem Lehrgang oder der ZAIM.
Korrespondenz:

Prof. Dr. med. Edouard Battegay, FACP

ZAIM-Stiftungsratspräsident

Direktor Klinik und Poliklinik

für Innere Medizin

UniversitätsSpital Zürich

Rämistrasse 100, RAE B 17

CH-8091 Zürich

Tel. 0442552400

edouard.battegay[at]usz.ch

www.my-zaim.ch
Eine gute und umfassende Ausbildung der Ärzte von heute ist der Garant einer qualitativ hochstehenden medizinischen Versorgung von morgen. Dabei ist es mit rein medizinischer Ausbildung nicht getan. Heutzutage ist für die Karriere neben medizinischem Wissen und klinischer Erfahrung auch Management- und Führungskompetenz gefordert. Um zukünftige Fachärzte für Allgemeine Innere Medizin (AIM) auf ihrem Karriereweg zu unterstützen, hat die Zurich Academy of Internal Medicine (ZAIM) den Lehrgang «KarriereEntwicklung Assistenzärzte» ins Leben gerufen. Die Teilnehmer des Lehrgangs sollen ihre Persönlichkeit, ihr Auftreten und ihre Resilienz stärken, sich erste Führungskompetenzen aneignen und ihren Berufsweg zielorientierter gestalten.

Die ZAIM verfolgt damit ihr Ziel, den Fortbestand einer hochstehenden Allgemeinen Innere Medizin zu fördern, indem sie junge Talente für die Disziplin AIM begeistert und mit dem notwendigen Rüstzeug für den entsprechenden Berufsweg ausstattet (siehe Kasten).

\section{Vorbereitung für eine Karriere in der Allgemeinen Inneren Medizin}

Anfang April 2014 fand das erste Modul des ersten Lehrgangs in Zürich statt. Unter dem Motto «Sie als Individuum» standen die Assistenzärzte mit ihrer Persönlichkeit im Zentrum. In dem aus Referaten, Workshops und Podiumsdiskussionen bestehenden zweitägigen Kurs analysierten die Teilnehmer ihre Chancen und Entwicklungsmöglichkeiten für eine Karriere in der Allgemeinen Inneren Medizin.

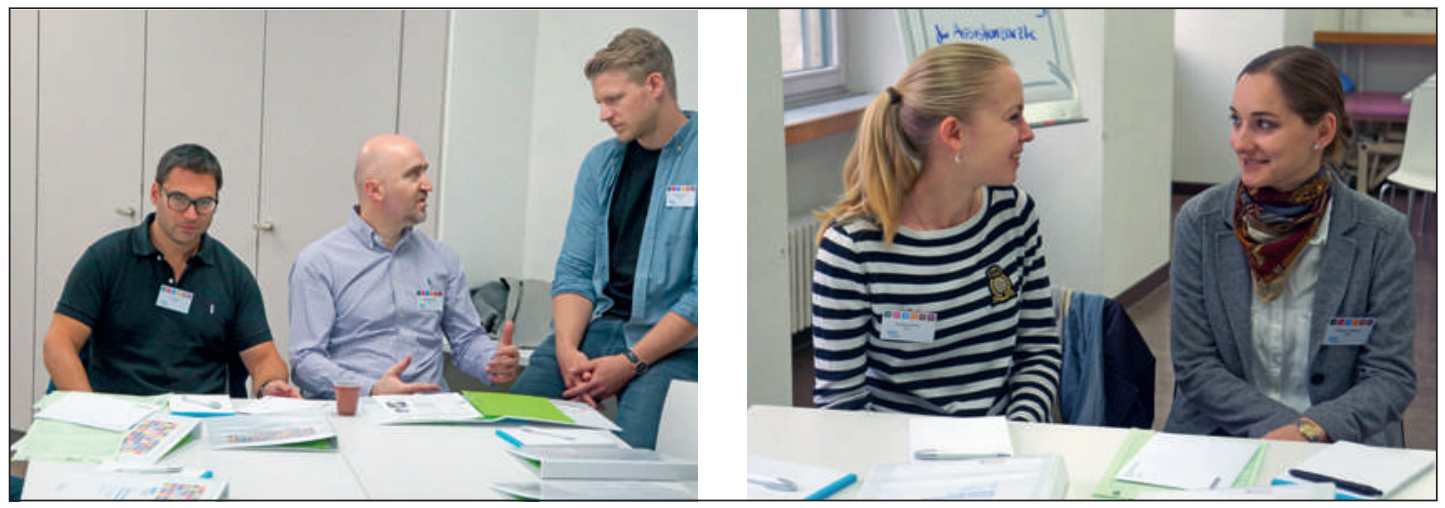

Die Teilnehmerinnen und Teilnehmer des ersten ZAIM-Lehrgangs tauschten sich unter anderem über Zeitmanagement und persönliche Ressourcen aus.

\section{Résumé}

La Zurich Academy of Internal Medicine (ZAIM) s'engage pour une Médecine Interne Générale de demain de haute qualité. Pour être en mesure de soutenir des futurs spécialistes de la Médecine Interne Générale tout au long de leur carrière, la ZAIM a initié le cours de formation «Développement de carrière des médecins-assistants». Ce cours de formation offre à ses participants la possibilité de renforcer leur personnalité, leur façon de se présenter et leur résilience, d'acquérir de premières compétences de direction et de mieux cibler leur parcours de carrière. Le premier module du tout premier cours de formation a eu lieu début avril 2014 à Zurich, le deuxième module suivra fin août 2014. Au printemps 2015 se déroulera le prochain cours de formation. On peut trouver des informations supplémentaires sur www.my-zaim.ch.

Rolf Zemp, selbständiger Berater, Coach und Fachhochschuldozent für Unternehmens- und Personalführung, diskutierte mit den Teilnehmern $\mathrm{Zu}$ kunftsperspektiven und künftige Anforderungen, insbesondere im Hinblick auf nicht-medizinische Kompetenzen wie Führungs- und Managementfähig- 


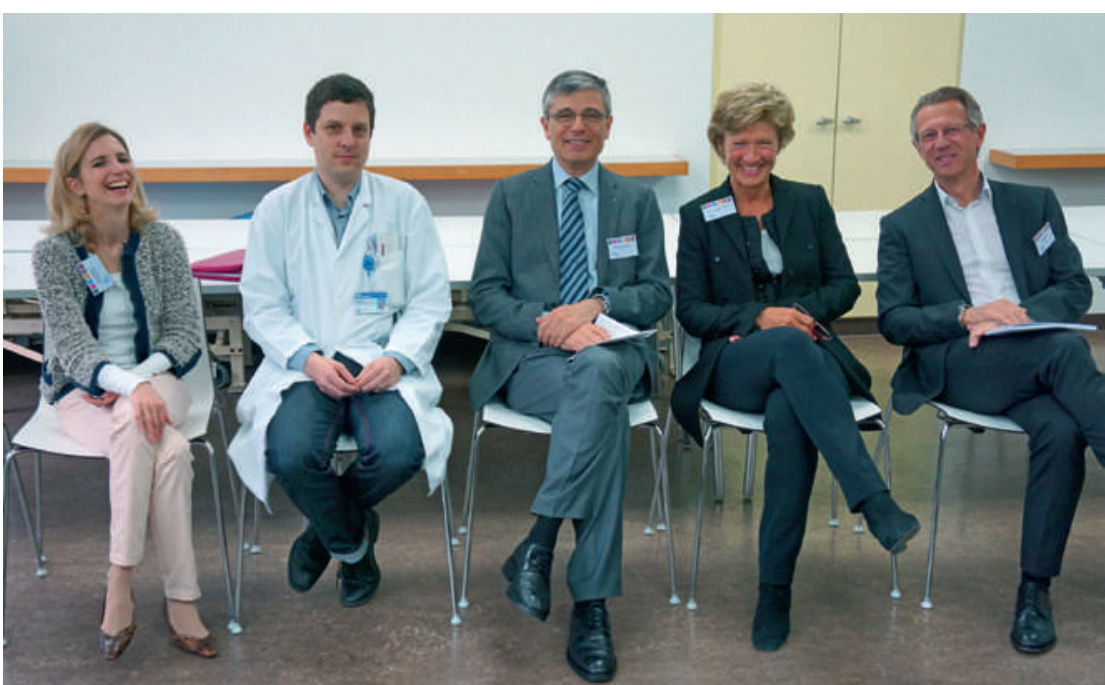

Engagement für Karriereplanung von Assistenzärztinnen und -ärzten: Franziska Gottschalk, Lukas Zimmerli, Edouard Battegay, Petra-Maria Schumm-Draeger, Rolf Zemp (v.I.n.r.)

keiten. Die Komplementarität zwischen medizinischem und wirtschaftlichem Denken und Handeln sollte akzeptiert und verinnerlicht werden.

Prof. Dr. med. Maria-Petra Schumm-Draeger, Chefärztin Klinik für Endokrinologie, Diabetologie und Angiologie am Klinikum Bogenhausen, Städtisches Klinikum München, gab einen Einblick in die richtige Karriereplanung. Maria-Petra Schumm-Draeger ist in der Kommission «Struktur-Krankenhausversorgung» der Deutschen Gesellschaft für Innere Medizin (DGIM) tätig und Delegierte der DGIM im Administrative Council der European Federation of Internal Medicine (EFIM) sowie aktives Mitglied der «Working Group on Professional Issues in Internal Medicine in Europe».

Laut Maria-Petra Schumm-Draeger sind die drei häufigsten karrierehinderlichen Faktoren geringe fachliche Laufbahnunterstützung, ungünstige $\mathrm{Ar}$ beitsplatzbedingungen und extraprofessionelle Belange wie Work-Life-Balance bzw. -Interference, z.B. die Vereinbarkeit von Beruf, Kinderbetreuung und Familie. Aber auch Faktoren bei Ärzten selbst wie Leistungsmotivation, Sozialkompetenz, Durchsetzungskraft und Flexibilität hätten grossen Einfluss auf die Karrierechancen. Darüber hinaus machte sie auf den hohen Stellenwert der Allgemeinen Inneren Medizin aufmerksam: In der stationären Versorgung fallen in Deutschland beinahe $40 \%$ aller Fälle in den Bereich der Inneren Medizin und 65\% dieser Fälle sind keinem spezifischen Schwerpunkt zugeordnet.

\section{Die eigene Persönlichkeit und individuelle Wirkung}

Für die persönliche Standortbestimmung führten die Teilnehmer vorab einen Persönlichkeitstest durch, dessen Ergebnis in Einzelgesprächen erörtert wurde. Franziska Gottschalk, Abteilungsleiterin Personalentwicklung \& Bildung des Human Resources Management am UniversitätsSpital Zürich, und ihr Team arbeiteten mit den Teilnehmern in kleinen Gruppen an deren Persönlichkeitsentwicklung sowie dem Selbst- und Zeitmanagement. Mittels individuellen Kameratrainings wurden zudem das Auftreten der Assistenzärzte und ihre Wirkung auf Dritte analysiert sowie Entwicklungspotentiale eruiert.

Dr. phil. et lic. iur. Mariann Grawe-Gerber und lic. phil. Misa Yamanaka vom Klaus-Grawe-Institut für Psychologische Therapie in Zürich gaben einen Einblick in das wichtige Thema Resilienz und persönliches Ressourcenmanagement. Nach der theoretischen Einführung konnten sich die Assistenzärzte in Zweiergesprächen über eigene Ressourcen sowie die Möglichkeiten deren Nutzung, aber auch über eigene Problembereiche und Ansatzpunkte zur Verbesserung bewusst werden.

\section{Der Assistenzarzt als Führungsperson}

Gemäss Aussagen der teilnehmenden Assistenzärzte trifft das Thema Karriereplanung den Nerv der Zeit. Die 18 Teilnehmer waren vom ersten Modul begeistert. Das zweite Modul befasst sich Ende August 2014 unter dem Motto «Sie als Führungsperson» und «Sie in Ihrem Umfeld» mit den Grundlagen der Führung in einem komplexen Umfeld: mit Führung ohne Vorgesetztenfunktion, mit Mitarbeiter-Typen, Führungstechniken und -stilen sowie mit Change-Management in einer Internistischen Klinik, mit juristischen Fragen wie Aufklärungspflicht, Dokumentation, Haftungsfragen, Arbeitsrecht und mit betriebswirtschaftlichen Zusammenhängen.

Der nächste Lehrgang «Karriere-Entwicklung Assistenzärzte» der Zurich Academy of Internal Medicine wird im Frühjahr 2015 stattfinden. Informationen hierzu und zu weiteren Themen der ZAIM finden sich auf der Website www.my-zaim.ch.

\section{ZAIM: Stiftung für eine qualitativ hochstehende Allgemeine Innere Medizin}

Die Zurich Academy of Internal Medicine (ZAIM) ist eine Stiftung, die es sich zum Ziel gesetzt hat, durch Weiter- und Fortbildung sowie Informationsaustausch sowie Forschung zu Themen ihres Fachgebietes eine qualitativ hochstehende Allgemeine Innere Medizin zu fördern. Angesichts der starken Spezialisierungstendenzen ist dies unerlässlich, um auch in Zukunft eine optimale medizinische Versorgung zu gewährleisten. Unser Gesundheitswesen braucht hochkompetente Allgemeininternisten im Interesse der Patienten und der Gesellschaft.

Der Stiftungsrat besteht aus Prof. Dr. med. Edouard Battegay (Präsident), PD Dr. med. Rubino C. Mordasini, Prof. Dr. iur. Urs Saxer und Prof. Dr. med. Petra-Maria Schumm-Draeger. 